\title{
Classification of Ultrasound Kidney Images using PCA and Neural Networks
}

\author{
Mariam Wagih Attia \\ Faculty of Computers \& Information Sciences, \\ Mansoura University, Mansoura, Egypt
}

F.E.Z. Abou-Chadi

Faculty of Engineering, The British University in Egypt, Cairo, Egypt

\begin{abstract}
In this paper, a computer-aided system is proposed for automatic classification of Ultrasound Kidney diseases. Images of five classes: Normal, Cyst, Stone, Tumor and Failure were considered. A set of statistical features and another set of multi-scale wavelet-based features were extracted from the region of interest (ROI) of each image and the principal component analysis was performed to reduce the number of features. The selected features were utilized in the design and training of a neural network classifier. A correct classification rate of $97 \%$ has been obtained using the multi-scale waveletbased features.
\end{abstract}

Keywords-Ultrasound kidney images; Feature Extraction; Principal Component Analysis; Neural Network classifier

\section{INTRODUCTION}

In recent years, great advances have been made in automated systems for detecting kidney diseases using ultrasonic systems which allow a greater amount and quality of information to be extracted during imaging the patients. The use of feature extraction, image analysis and pattern recognition techniques for classification is most suited to the evaluation of global conditions (e.g. failure, stone, tumor, and cyst).

Previous work have utilized feature extraction techniques such as Gray Level Statistical features, Fourier Transform and Gabor Wavelet's features with kidney and liver ultrasound images [1-3].

The present paper describes an automated system for analyzing and classifying ultrasound kidney images. The system starts with capturing the ultrasound kidney image and identifying the region of interest. Image Preprocessing techniques are also employed to improve image quality and reduce noise. Discrete Wavelet Transform (DWT) was used for feature extraction as it has potential capacity in classification problems [4]. Moreover, statistical features were extracted for the comparison purposes. Feature extraction with DWT yields to a large number of features being extracted, so the PCA technique was employed as being efficient in selecting the optimal features [1], [5], and [6]. The last stage in the system is the classifier. A multi-layer neural network was designed and trained using the optimal features selected by PCA.

\author{
Hossam El-Din Moustafa \\ Faculty of Engineering, \\ Mansoura University, Mansoura, Egypt \\ Nagham Mekky \\ Faculty of Computers \& Information Sciences, \\ Mansoura University, Mansoura, Egypt
}

The characteristics of the designed classifier were investigated and optimized for both best performance and highest classification rate [7]. Fig. 1 shows a block diagram for the proposed system.

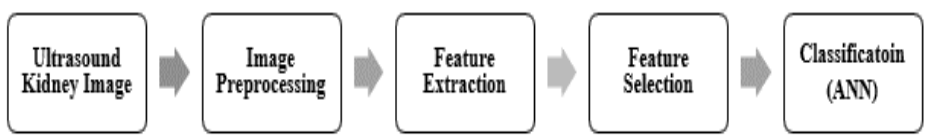

Fig. 1. The Proposed system for automatic classification of kidneys images

The paper is organized as follows: Section II describes the database used and the kidney diseases considered in the present study. Image preprocessing techniques are explained in Section III. Sections IV and V present the feature extraction and feature selection techniques utilized, respectively. The classification stage is explained in Section VI where an artificial neural network is utilized. Section VII gives the concluding remarks.

\section{DATA COLLECTION}

Sixty-Six ultrasound kidney images were obtained from ULTRASCAN CENTRE - Ernakulum, Cochin, Kerala, India (http://www.ultrasound-images.com/kidneys.htm) and Ultrasound Guide for Emergency Physicians-Johns Hopkins University Department of Emergency Medicine (http://www.sonoguide.com/renal.html) ; it includes a collection of images for normal kidney and kidney with multiple kinds of diseases [8] such as:

- Angiomyolipomas (Tumor): These are the most common benign tumor of the kidney and are composed of blood vessels, smooth muscle cells and fat cells.

- Renal failure: It is a medical condition in which the kidneys fail to adequately filter waste products from the blood.

- Cystic kidney disease: it is a cystic genetic disorder of the kidneys

- Kidney Stones: Kidney stones come in different varieties such as, Calcium-containing stones, Uric acid stones, or infected stones. 
TABLE I. TYPES OF DISEASES AND THE NUMBER OF IMAGES USED FOR EACH TYPE

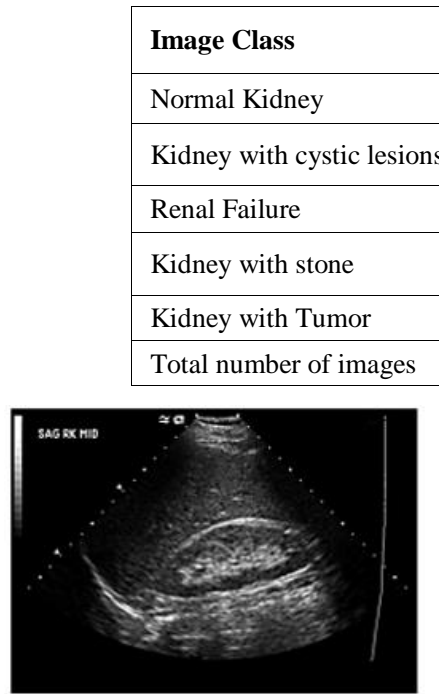

(a)

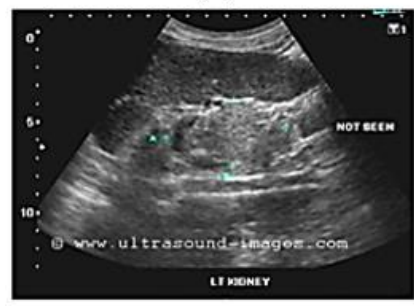

(c)

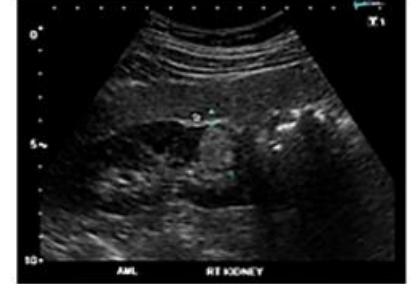

(b)

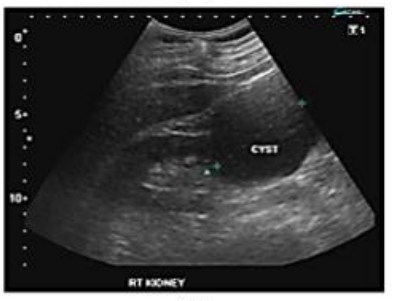

(d)

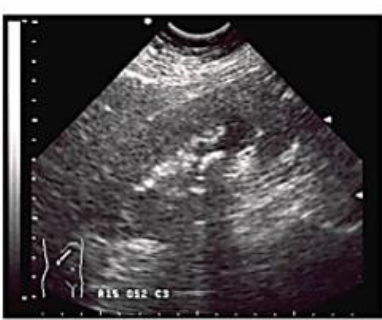

(e)

Fig. 2. Kidney ultrasound images (a) Normal, (b) Kidney with tumor, (c) Kidney failure, (d) Kidney with cystic lesions, and (e) kidney with stone

\section{IMAGE PREPROCESSING}

\section{A. Region-Of-Interest (ROI)}

The first step in image preprocessing is to determine the region of interest (ROI). It will improve the speed and accuracy of classification process by selecting only the kidney and removing unneeded details like patient and scan information. Previous researches have proposed automated ways to get the ROI [9] nevertheless, in the present work, a rectangular ROI of size 256x256 was obtained manually by cropping the kidney image to simplify the process and limit the possibilities of errors. The ROI size of 256x256 pixels was chosen as being suitable for both longitudinal and transverse kidney images. Fig .3 shows a normal kidney image with outlined ROI area.

\section{B. Speckle noise}

To improve the quality of ultrasound kidney images, image-preprocessing techniques have been adopted. Three filters were applied and their performance was compared and evaluated in terms of entropy. These are: Wiener, Histogram Equalization and Median Filter.

\section{1) Wiener Filter}

Wiener filter is used to reduce the noise present in the image [10]. Fig. 4 shows the output image after using Wiener filter.

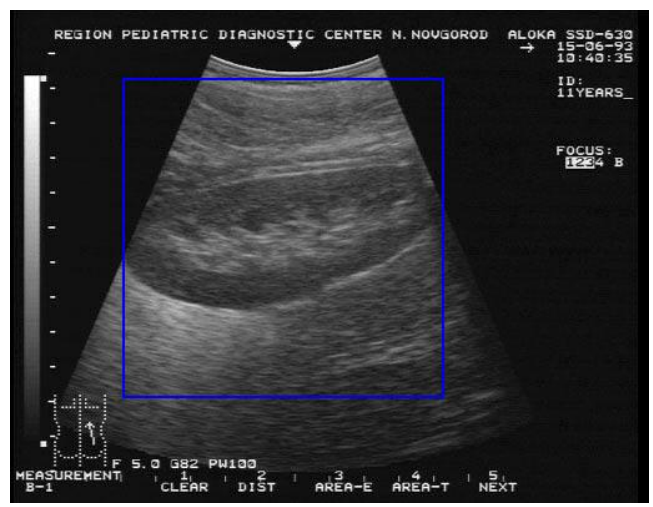

Fig. 3. Normal Kidney Image with outlined ROI

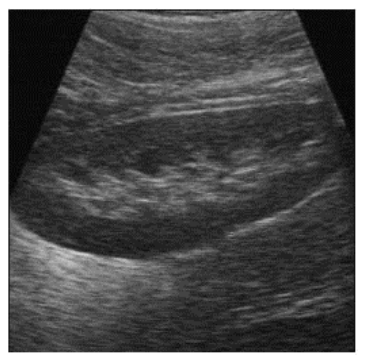

(a)

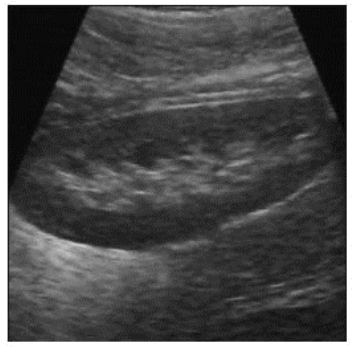

(b)
Fig. 4. ROI of Kidney image (a) Original Image, (b) Wiener filtered image

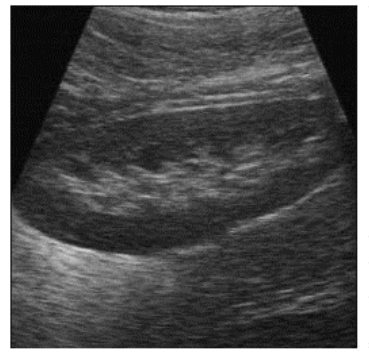

(a)

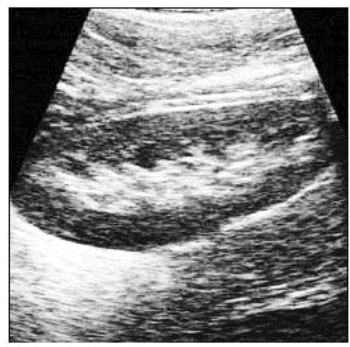

(b)
Fig. 5. ROI of Kidney image (a) Original Image, (b) output of histogram equalization 


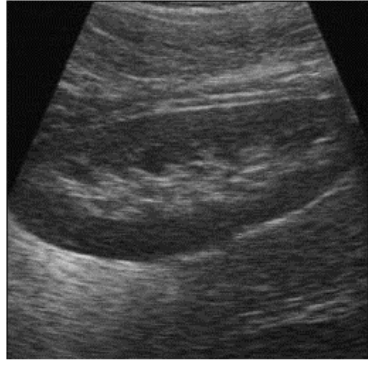

(a)

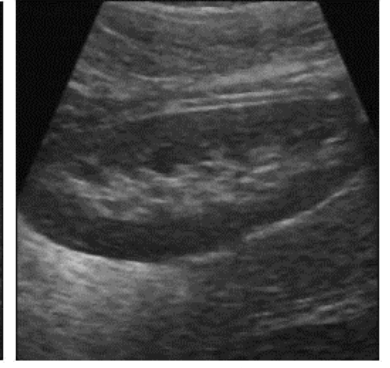

(b)
Fig. 6. ROI (a) Original Image, (b) output of Median Filter

\section{2) Histogram Equalization}

Histogram equalization is used to improve the visual appearance of an image by adjusting the image histogram [11],[12] . Fig. 5 shows the results of applying histogram equalization.

\section{3) Median Filter}

Median Filter helps in reducing mainly speckle and salt and pepper noise [12]. Fig. 6 shows the resulting image after using a $3 \times 3$ median filter.

The results showed that Median filter gives the best performance especially if the evaluator is concerning more on the kidney edges than the whole image. This is in agreement with that reported in [11]

\section{FEATURE EXTRACTION}

\section{A. Statistical Features}

Statistical features is one of the early methods proposed in image processing. The gray level co-occurrence matrix (GLCM) of the ROI was used as suggested by Haralick [13].

The following features are extracted from the GLCM of the ROI kidney images using MATLAB: Energy, Entropy, Contrast, Homogeneity, Maximum probability and correlation [14].

1) Energy is a measure of local homogeneity and it is calculated using:

Energy $=\frac{1}{M N} \sum_{j=1}^{M} \sum_{i=1}^{N}\{X(i, j)\}^{2}$

where, $i$ and $j$ are the pixel values.

2) Entropy measures the average, global information content of an image in terms of average bits per pixel. As the magnitude of entropy increases, more information is associated with the image.

Entropy $=-\sum_{i} \sum_{j} p(i, j) \log p(i, j)$

3) Contrast defines the difference between the lightest and darkest areas on an image.

Contrast $=\sum_{i} \sum_{j}(i-j)^{2} p(i, j)$

4) Homogeneity is the state or quality of being homogeneous, biological or other similarities within a group.

Homogeneity $=\sum_{i} \sum_{j} \frac{p(i, j)}{1+|i-j|}$
5) Correlation is a measure of the strongest of the relationship between two variables.

Correlation $=\frac{\operatorname{cov}(x, y)}{\sigma_{x} \sigma_{y}}$

Table II depicts the results of feature extraction associated with each class of images. Values in the upper row are the mean values for each class and the lower row gives the standard deviation for each class.

\section{B. Discrete wavelet Transform}

The discrete wavelet transform (DWT) is a multi-resolution analysis technique that analyzes the signal by decomposing the signal into its coarse and detail information, this is accomplished by using successive high-pass and low-pass filtering operations [15], [16], based on following equations:

$$
\begin{aligned}
& y_{\text {high }}[k]=\sum_{n=-\infty}^{+\infty} x(n) \cdot g(2 k-n) \\
& y_{\text {low }}[k]=\sum_{n=-\infty}^{+\infty} x(n) \cdot h(2 k-n)
\end{aligned}
$$

TABLE II. Statistical Features Results

\begin{tabular}{|l|l|l|l|l|l|}
\hline \multirow{2}{*}{ Features } & \multicolumn{5}{c|}{ Image Classes } \\
\cline { 2 - 6 } & Normal & Failure & Stone & Tumor & \multirow{2}{*}{ Cyst } \\
\hline \multirow{2}{*}{ Entropy } & 5.695 & 5.990 & 5.260 & 6.129 & 6.472 \\
& 0.640 & 0.450 & 0.964 & 0.110 & 0.773 \\
\hline \multirow{2}{*}{ Contrast } & 0.109 & 0.155 & 0.196 & 0.097 & 0.148 \\
& 0.025 & 0.021 & 0.021 & 0.010 & 0.047 \\
\hline \multirow{2}{*}{ Correlation } & 0.942 & 0.900 & 0.935 & 0.928 & 0.954 \\
& 0.008 & 0.031 & 0.009 & 0.003 & 0.020 \\
\hline \multirow{2}{*}{ Energy } & 0.304 & 0.282 & 0.358 & 0.293 & 0.239 \\
& 0.086 & 0.065 & 0.136 & 0.029 & 0.097 \\
\hline \multirow{2}{*}{ Homogenety } & 0.959 & 0.948 & 0.945 & 0.955 & 0.942 \\
& 0.004 & 0.008 & 0.009 & 0.004 & 0.012 \\
\hline
\end{tabular}

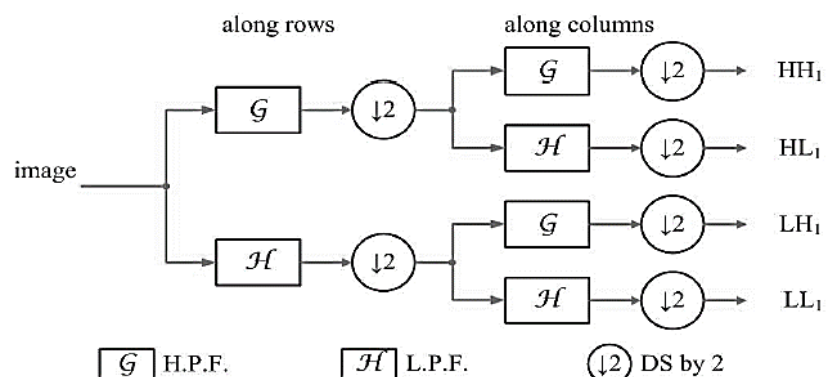

Fig. 7. One level of the DWT transform for 2D signal

\section{Feature SELECTION}

The Principal Component Analysis (PCA) technique was used frequently in previous work for feature reduction in classification problems with ultrasound images [1], [5]. PCA was also used in conjunction with DWT in other object classification problems like face recognition [17]. In this work, PCA helps in reducing the feature vector dimension obtained from DWT of ultrasound kidney images.

Procedure for making PCA:

- Getting the covariance matrix.

- Getting the Eigen Vectors

- Selecting top Eigen Values from Eigen Vectors 
Using MATLAB, the first step in this procedure results in a covariance matrix of size $32 \times 32$. Second step gives an eigenvector of 32 values i.e. the eigenvalues or the principal components .

The results have shown that the first 15 eigenvalues in the eigenvector, can be considered as useful-nonzero values. These first 15 values will then be used in the classification stage.

\section{NEURAL NETWORK ClaSSIFICATION}

\section{A. Neural Network Topology Design}

Using "NeuralBuilder" module in "NeuroSolutions" software provided by "NeuroDimension", Inc., a multilayer neural network as shown in Fig. 8, with two hidden layers with 10 nodes each was designed. Five output nodes were used to produce the following output encoding for the five kidney image classes- ' 10000 ' for 'Normal', '01000' for 'Failure', '00100' for 'Stone', '00010' for 'Tumor' and '00001' for 'Cyst'. A Sanger's rule and sigmoidal activation function were found suitable for the classification purpose. A mean square error value of 0.05 was used to stop the learning process.

\section{B. Building and Training ANN}

Network characteristics like number of hidden layers, processing elements in each layers, optimization method and learning rule are customizable and could be adjusted for getting better learning rate and less mean square error (MSE) which is an important measure of network performance. Different topologies of neural networks were used to reach the best results.

Optimal results were obtained when the number of processing elements in the first hidden layer is 3 nodes and in the second hidden layer is 6 nodes.

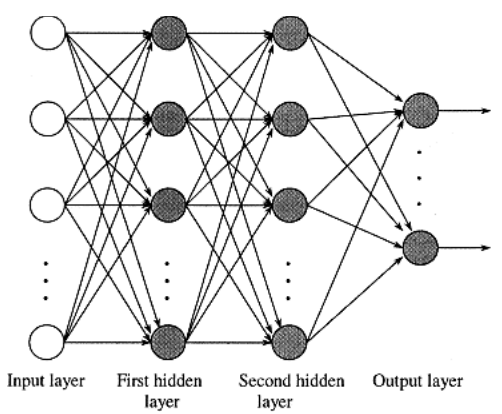

Fig. 8. Multilayer ANN with two hidden layers

Training was performed using the hold-out method where $50 \%$ of the data were used for training the classifier and $50 \%$ for testing [18]. Each set of features was used separately and the results were compared.

A correct classification rate of $95 \%$ was obtained using statistical features; on the other hand, $97 \%$ of the images were correctly classified when trained with wavelet features. Table III illustrates the results for each feature set; where sensitivity and specificity were calculated using equations (9), (10) respectively.

Sensitivity = true positives $/($ true positive + false negative $)$
Specificity = true negatives $/($ true negative + false positives $)$

\section{CONCLUSION}

In this paper, an automatic system for the detection and classification of kidney diseases has been developed. The system consists of five main parts: ROI segmentation, image preprocessing, feature extraction, feature selection and classification. ROI segmentation was performed manually with the help of the physician by cropping. Image preprocessing was carried out using three types of filters: Wiener filter, Median filter and Histogram Equalization filter. The results showed that Median filter gives the best performance. Two sets of features were extracted using two different features extraction techniques. These are statistical-based features and the multi-scale wavelet-based features. Feature selection was achieved using the principal component analysis approach. A multilayer feed forward neural network utilizing the backpropagation algorithm was used for classification purpose. It has been shown that the highest classification rate was obtained using the multi-scale wavelet-based features. A correct classification rate of $97 \%$ has been obtained which is comparable to similar neural networks classifiers used in [1], [2], [19]. The results are encouraging and promising. Further work is required to apply the suggested methodologies to a larger data set with a wide spectrum of kidneys disorders and to develop a complete intelligent system that can be used as an assistant tool in automatic classification of ultrasound kidney images. Improving the classification accuracy is a subject of a current investigation which aims to develop a complete automatic kidney images classification.

TABLE III. COMPARISON OF THE ClASSIFICATION RESULTS

\begin{tabular}{|c|c|c|c|c|}
\hline \multirow{3}{*}{$\begin{array}{l}\text { Image } \\
\text { Class }\end{array}$} & \multirow{2}{*}{\multicolumn{2}{|c|}{$\begin{array}{l}\text { Statistical Features } \\
\text { Classification Rate } \\
{[57 / 60](95 \%)}\end{array}$}} & \multirow{2}{*}{\multicolumn{2}{|c|}{$\begin{array}{l}\text { Wavelet- based Features } \\
\text { Classification Rate } \\
{[59 / 60](97 \%)}\end{array}$}} \\
\hline & & & & \\
\hline & Sensitivity & Specificity & Sensitivity & Specificity \\
\hline Normal & $\begin{array}{l}{[10 / 10]} \\
(100 \%)\end{array}$ & $\begin{array}{l}{[48 / 50]} \\
(96 \%)\end{array}$ & $\begin{array}{l}{[10 / 10]} \\
(100 \%)\end{array}$ & $\begin{array}{l}{[50 / 50]} \\
(100 \%)\end{array}$ \\
\hline Failure & $\begin{array}{l}{[10 / 10]} \\
(100 \%)\end{array}$ & $\begin{array}{l}{[50 / 50]} \\
(100 \%)\end{array}$ & $\begin{array}{l}{[10 / 10]} \\
(100 \%)\end{array}$ & $\begin{array}{l}{[50 / 50]} \\
(100 \%)\end{array}$ \\
\hline Stone & $\begin{array}{l}{[10 / 12]} \\
(83 \%)\end{array}$ & $\begin{array}{l}{[48 / 48]} \\
(100 \%)\end{array}$ & $\begin{array}{l}{[11 / 12]} \\
(91 \%)\end{array}$ & $\begin{array}{l}{[48 / 48]} \\
(100 \%)\end{array}$ \\
\hline Tumor & $\begin{array}{l}{[10 / 10]} \\
(100 \%)\end{array}$ & $\begin{array}{l}{[49 / 50]} \\
(98 \%)\end{array}$ & $\begin{array}{l}{[10 / 10]} \\
(100 \%) \\
\end{array}$ & $\begin{array}{l}{[49 / 50]} \\
(98 \%) \\
\end{array}$ \\
\hline Cyst & $\begin{array}{l}{[17 / 18]} \\
(94 \%)\end{array}$ & $\begin{array}{l}{[52 / 52]} \\
(100 \%)\end{array}$ & $\begin{array}{l}{[17 / 18]} \\
(94 \%)\end{array}$ & $\begin{array}{l}{[52 / 52]} \\
(100 \%)\end{array}$ \\
\hline
\end{tabular}

[1] Balasubramanian, D., Coll. of Eng., Chennai, Srinivasan, P., Gurupatham, R. "Automatic classification of focal lesions in ultrasound liver images using principal component analysis and neural networks," Engineering in Medicine and Biology Society, 2007. EMBS 2007. 29th Annual International Conference of the IEEE, Lyon, France, pp. 2134 - 2137, August 2007.

[2] K.Bommanna Raja, M.Madheswaran and K.Thyagarajah "Analysis of Ultrasound kidney Images using Content Descriptive Multiple Features for Disorder Identification and ANN based Classification," International Conference on Computing: Theory and Applications 2007 (ICCTA'07), pp. $382-388$

[3] Hafizah, W.M., Supriyanto, E., Yunus, J. "Feature Extraction of Kidney Ultrasound Images Based on Intensity Histogram and Gray 
Level Co-occurrence Matrix” Modelling Symposium (AMS), 2012 Sixth Asia, pp. 115 - 120

[4] Yu-Long Qiao, Harbin ; Chun-Yan Song and Chun-Hui Zhao "DoubleDensity Discrete Wavelet Transform Based Texture Classification" Intelligent Information Hiding and Multimedia Signal Processing, 2007. IIHMSP 2007. Third International Conference on (Volume:1 ), pp. 91 94.

[5] Jiř́i Blahuta, Tomáš Soukup and Petr Čermák "The image recognition of brain-stem ultrasound images with using a neural network based on PCA" International Journal Of Applied Mathematics and Informatics Issue 2, Volume 5, 2011, pp. 46 - 54.

[6] Chris Ding and Xiaofeng He, "K-Means Clustering via Principal Component Analysis", In proceedings of the 21 st International Conference on Machine Learning, Banff, Canada, 2004 Page 29

[7] Bernd Jähne, Horst Haußecker, Peter Geißler "Handbook of Computer Vision and Applications" Volume 2, Signal Processing and Pattern Recognition 1999, pp. 275-308

[8] Harald Lutz, Elisabetta Buscarini "WHO manual of diagnostic ultrasound. Vol. 1. -- 2nd ed" World Health Organization 2011, Chapter 13 Kidneys and ureters

[9] Wan Mahani Hafizah, Eko Supriyanto, "Automatic Region of Interest Generation for Kidney Ultrasound Images " ACS'11 Proceedings of the 11th World Scientific and Engineering Academy and Society (WSEAS) international conference on Applied computer science Stevens Point, Wisconsin, USA, 2011, pp. 70-75

[10] Brown, Robert Grover; Hwang, Patrick Y.C.. "Introduction to Random Signals and Applied Kalman Filtering (3 ed.)". New York: John Wiley \& Sons (1996). ISBN 0-471-12839-2.

[11] Wan Mahani Hafizah, Eko Supriyanto, "Comparative Evaluation of Ultrasound Kidney Image Enhancement Techniques" , International
Journal of Computer Applications (0975 - 8887) Volume 21- No.7, May 2011.

[12] Shradha Dakhare, Harshal Chowhan, Manoj B.Chandak "Combined Approach for Image Segmentation" International Journal of Computer Trends and Technology (IJCTT), Volume 11 No. 3, May 2014, pp. 118 $-121$.

[13] K. Shanmugam R. M. Haralick and I. H. Dinstein, "Textural features for image classification" IEEE Transactions on Systems, Man and Cybernetics 3 (1973), pp. 610 - 621.

[14] S. Manikandan, V. Rajamani, "A Mathematical Approach for Feature Selection \& Image Retrieval of Ultra Sound Kidney Image Databases", European Journal of Scientific Research ISSN 1450-216X Vol.24 No.2 (2008), pp.163-171

[15] Carmen Mariana Nicolae and Luminita Moraru "Image Analysis of Kidney Using Wavelet Transform" Annals of the University of Craiova, Mathematics and Computer Science Series, Volume 38(1), 2011, pp. 27-34, ISSN: 1223-6934

[16] G. Simone, F.C. Morabito, R. Polikar, P. Ramuhalli, L. Udpa and S. Udpa "Feature extraction techniques for ultrasonic signal classification" International Journal of Applied Electromagnetics and Mechanics 15 (2001/2002) pp. 291-294.

[17] Mrs.N.G.Chitaliya, Prof.A.I.Trivedi "Feature Extraction using WaveletPCA and Neural network for application of Object Classification \& Face Recognition" Second International Conference on Computer Engineering and Applications 2010, pp. 510-514

[18] C. M. Bishop. "Neural Networks for Pattern Recognition." Oxford University Press, 1995.

[19] Deepika Gupta, Ajay Kumar Singh, Deepa Kumari, Raina "Hybrid Feature based Natural Scene Classification using Neural Network" International Journal of Computer Applications (0975 - 8887) Volume 41- No.16, March 2012, pp. 48 - 52. 